\title{
Infestation of the eyelashes with Phthirus pubis
}

\author{
Dong-Lai Ma MD, Sergio Vano-Galvan MD
}

Previously published at www.cmaj.ca

A 36-year-old woman presented with a three-week history of persistent itching of the eyelids of her right eye. Her husband had had pubic pruritus for about one month. Examination revealed a few lice and nits at the roots of the woman's eyelashes (Figure 1). The right conjunctiva appeared reddish, but the eye was otherwise normal. Numerous lice and nits were also seen in the pubic area. All the other hair-bearing skin, including the eyebrows, axillae, body hair and scalp, were inspected for lice and nits, neither of which were seen. The specimen proved microscopically to be Phthirus pubis and their nits (Appendix 1, available at www.cmaj.ca/cgi/content/full/cmaj .090793/DC1). A screen for other sexually transmitted infections, including serologic testing for HIV, syphilis and hepatitis B and C, showed no abnormality.

The lice were removed from the eyelashes with fine forceps, and petrolatum was applied twice daily. The pubic area was shaved and treated with $10 \%$ sulfur ointment once daily for three days. The patient was advised to wash all clothing and fomites. Her husband was treated simultaneously. Two weeks later, the itching resolved, and the eyelashes and pubic area remained free of parasites.

Although $P$. pubis can infest any hair-bearing area, infestation of the eyelashes (phthiriasis palpebrum) is uncommon in healthy adults. All hair-bearing areas should be examined carefully if infestation is found in one area. Because $P$. pubis in adults is frequently transmitted sexually, a screen for other sexually transmitted infections should be done. In children, eyelashes are the most common site of infestation. ${ }^{1}$ Children are usually infected through direct passage of the lice from their parents or other infected contacts. ${ }^{2}$

The goal of therapy is to eliminate the lice and eggs. All contacts should be treated simultaneously. Treatment options for infestation of the eyelashes include $1 \%$ permethrin lotion or petrolatum. ${ }^{3}$ Petrolatum is effective in eradicating lice, but it does not destroy the eggs. It should be applied twice daily for 8 to 10 days. $^{3}$ Lindane lotion and oral therapy with ivermectin have also been used. ${ }^{4}$ Pediculosis pubis can also be

From the Department of Dermatology, Peking Union Medical College Hospital, Chinese Academy of Medical Sciences and Peking Union Medical College (Ma), Beijng, China; and the Department of Dermatology, Hospital Ramon y Cajal (Vano-Galvan), Madrid, Spain

CMAJ 2010. DOI:10.1503/cmaj.090793

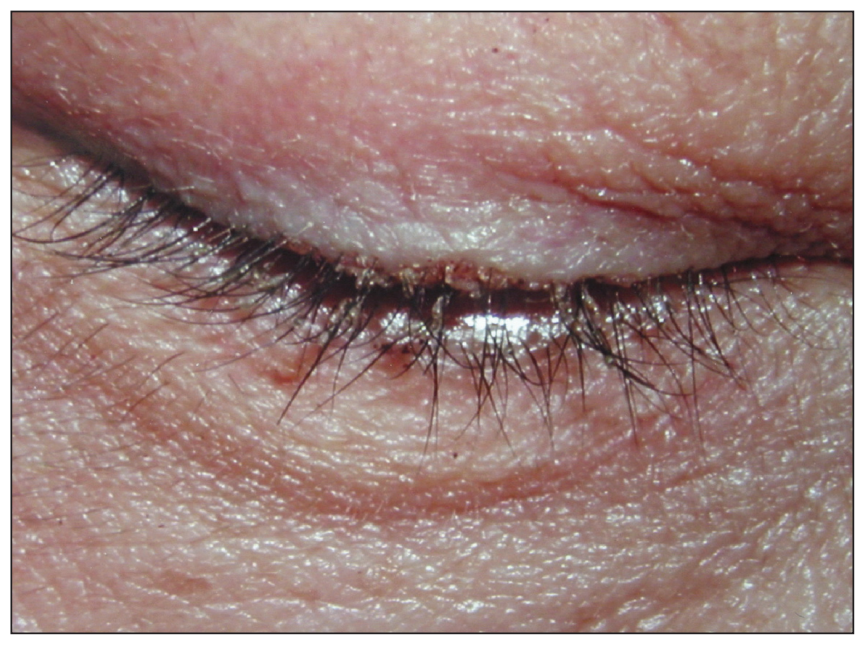

Figure 1: Phthirus pubis and nits attached to the roots of the eyelashes of a 36-year-old woman.

treated with these agents, as well as malathion and phenothrin lotions. ${ }^{3}$ Patients with $P$. pubis infestation should be checked seven days after treatment has begun. If the infestation has not been eradicated, another therapy should be started.

Experts recommend that clothing, towels and bedding used by the patient within two to three days before treatment began should be machine washed (with water at least $55^{\circ} \mathrm{C}$ ) and dried on the hot cycle for 5-10 minutes. Items that cannot be washed can be dry cleaned or stored in a sealed plastic bag for two weeks. ${ }^{5}$

This article has been peer reviewed.

Competing interests: None declared.

\section{REFERENCES}

1. Burgess IF. Human lice and their management. Adv Parasitol 1995;36:271-342.

2. Ikeda N, Nomoto H, Hayasaka S, et al. Phthirus pubis infestation of the eyelashes and scalp hairs in a girl. Pediatr Dermatol 2003;20:356-7.

3. Clinical Effectiveness Group. United Kingdom national guideline on the management of Phthirus pubis infestation. London (UK): British Association of Sexual Health and HIV; 2008. Available: www.bashh.org/documents/28/28.pdf (accessed 2009 June 9).

4. Burkhart CN, Burkhart CG. Oral ivermectin therapy for phthiriasis palpebrum. Arch Ophthalmol 2000;118:134-5.

5. Ngai JW, Yuen HK, Li FC. An unusual case of eye itchiness. Hong Kong Med J 2008;14:414-5. 